\title{
TOPOLOGICAL SPACES THAT ARE $\alpha$-FAVORABLE FOR A PLAYER WITH PERFECT INFORMATION
}

\author{
H. E. WHITE, JR.
}

ABSTRACT. The class of spaces mentioned in the title is closely related to the class of $\alpha$-favorable spaces introduced by G. Choquet [3]. For convenience, call the spaces mentioned in the title weakly a-favorable. The following statements are true: (1) every dense $G_{\delta}$ subset of a quasi-regular, weakly $a$-favorable space is weakly $a$-favorable; (2) the product of any family of weakly $a$-favorable spaces is weakly $a$-favorable; (3) any continuous, open image of a weakly $\alpha$-favorable space is weakly $a$-favorable; (4) a quasi-regular space with a $\sigma$-disjoint pseudobase is weakly $a$-favorable if and only if it is pseudo-complete in the sense of J. C. Oxtoby; and (5) the product of a weakly $\alpha$-favorable space and a Baire space is a Baire space.

1. Introduction. In recent years, a number of classes of topological spaces have been considered, each of which is a subclass of the class of Baire spaces, and each of which is closed under the formation of products (see [1] for a discussion of these classes). The purpose of this note is to show that the class of spaces mentioned in the title has a number of reasonable properties. The author would like to thank D. J. Lutzer for suggesting (in a letter) the desirability of finding a class of spaces satisfying statements which are essentially (2), (4), (5), (6), (7), (8), and (11) of the theorem, and for supplying the author with a copy of [1], which proved very helpful.

2. Definitions. For any function $\phi$, let $D(\phi), R(\phi)$ denote the domain and the range of $\phi$, respectively. For any collection $K$ of sets, let $K^{*}=K \sim\{\varnothing\}$.

A topological space $(X, \mathcal{T})$ is called weakly $\alpha$-favorable (or $\alpha$-favorable for a player with perfect information) if there is a sequence $\mathcal{S}=\left(\phi_{n}\right)_{n \in N}$ of functions such that

(2.1) $D\left(\phi_{1}\right)=\mathcal{T}^{*} \supset R\left(\phi_{1}\right)$ and $\phi_{1}(U) \subset U$ for all $U$ in $D\left(\phi_{1}\right)$, (2.2) for all $n$ in $N$,

Received by the editors November 14, 1973 and, in revised form, April 10, 1974. AMS (MOS) subject classifications (1970). Primary 54E99, 54D99; Secondary 54E25, 54C10.

Key words and phrases. Weakly $a$-favorable, $\alpha$-favorable, pseudo-complete, $\sigma$-disjoint pseudo-base. 


$$
\begin{aligned}
D\left(\phi_{n+1}\right)=\left\{\left(U_{1}, \cdots, U_{n+1}\right) \in\left(\mathcal{J}^{*}\right)^{n+1}:\right. & \\
& \left.U_{j+1} \subset \phi_{j}\left(U_{1}, \cdots, U_{j}\right) \text { for } j=1, \cdots, n\right\},
\end{aligned}
$$

$R\left(\phi_{n+1}\right) \subset \mathcal{I}^{*}$, and $\phi_{n+1}\left(U_{1}, \cdots, U_{n+1}\right) \subset U_{n+1}$ for all $\left(U_{1}, \cdots, U_{n+1}\right)$ in $D\left(\phi_{n+1}\right)$, and

(2.3) if $\left(U_{n}\right)_{n \in N}$ is a sequence such that

$$
\left(U_{1}, \cdots, U_{n}\right) \in D\left(\phi_{n}\right) \text { for all } n \text { in } N,
$$

then $\bigcap\left\{U_{n}: n \in N\right\} \neq \varnothing$.

There is an interesting discussion in [3, pp. 115-116] which can be used to give an interpretation, in the language of game theory, of the preceding definition.

Any sequence $\mathcal{S}=\left(\phi_{n}\right)_{n \in N}$ which satisfies (2.1), (2.2), and (2.3) is called a winning strategy for $(X, \mathcal{T})$. A sequence $\left(U_{n}\right)_{n} \in N$ which satisfies (2.3.1) is called an $\mathcal{S}$-sequence.

A subfamily $\mathcal{P}$ of $\mathcal{T}$ is called a pseudo-base for $\mathcal{T}$ if every nonempty element of $\mathcal{T}$ contains a nonempty element of $\mathcal{P}$. A pseudo-base $\mathcal{P}$ is called $\sigma$-disjoint if $\mathcal{P}=\bigcup\left\{\mathscr{P}_{n}: n \in N\right\}$, where each $\mathscr{P}_{n}$ is a disjoint family.

3. Theorem. Suppose $(X, \mathfrak{T})$ is a topological space.

(1) If $X$ is weakly a-favorable, then $X$ is a Baire space.

(2) If $X$ is locally weakly $\alpha$-favorable, then $X$ is weakly a-favorable.

(3) If $X$ is either pseudo-complete [4] or a-favorable [3], then $X$ is weakly a-favorable.

(4) If, for each $i$ in $I, X_{i}$ is weakly $\alpha$-favorable and $m \geq \boldsymbol{N}_{0}$, then the $\mathrm{m}$ box product of $\left(X_{i}\right)_{i \in I}$ is weakly a-favorable.

(5) If $X$ is weakly $\alpha$-favorable and $U$ is open in $X$, then $U$ is weakly $\alpha$ favorable.

(6) If $X$ is quasi-regular [4] and weakly $\alpha$-favorable, and $X_{0}$ is a dense $G_{\delta}$ subset of $X$, then $X_{0}$ is weakly a-favorable.

(7) If $\theta$ is a continuous, closed, irreducible mapping of $(X, \mathcal{J})$ onto $(Y, \mathcal{U})$, then $X$ is weakly a-favorable if and only if $Y$ is weakly a-favorable.

(8) If $\theta$ is a continuous, open mapping of $(X, \mathcal{J})$ onto $(Y, \mathcal{U})$, and $X$ is weakly a-favorable, then $Y$ is weakly $\alpha$-favorable.

(9) If $X$ is a Baire space and $(Y, \mathcal{U})$ is weakly a-favorable, then $X \times Y$ is a Baire space.

(10) If $X$ has a $\sigma$-disjoint pseudo-base $\mathcal{P}$, then $X$ is weakly $\alpha$-favorable if and only if it is a-favorable. If $X$ is also quasi-regular, then $X$ is weakly $\alpha$-favorable if and only if $X$ is pseudo-complete. 
(11) If $X$ is a $T_{0}$ space with a base of countable order [5], then $X$ is weakly $\alpha$-favorable if and only if there is a dense $G_{\delta}$ subset which is metrically topologically complete.

Remarks. (i) It follows from (10) that the concepts of weakly $\alpha$-favorable, $\alpha$-favorable, and pseudo-complete coincide for the class of quasiregular spaces which have dense metrizable subspaces. In particular, they coincide for quasi-regular, semi-metrizable spaces (since every semi-metrizable Baire space has a dense metrizable subspace).

(ii) It follows from (4) and (8) that $X \times Y$ is weakly $\alpha$-favorable if and only if both $X$ and $Y$ are weakly $\alpha$-favorable.

(iii) Statement (9) generalizes 4.2 of [2]. The proof given here is shorter and simpler than the proof of 4.2 that is given in [2].

(iv) Statement (11) is very similar to the corollary to Theorem 2.4 of [2].

(v) A generalization of Theorem 2.4 of [2] can be obtained by combining (3), (8), and (10).

Proof. The proofs of (1), (2), (3), and (5) are easy and are omitted. The proof of (4) is quite similar to the proof of theorem 7.12(iv) of [3], and is omitted.

(6) Suppose $\mathcal{S}=\left(\phi_{n}\right)_{n \in N}$ is a winning strategy for $(X, \mathcal{T})$. Since $\mathcal{T}$ is quasi-regular, the family $\mathcal{R}$ of all regular elements of $\mathcal{J}$ is a pseudo-base for $\mathcal{T}$; hence we may assume that $\bigcup\left\{R\left(\phi_{n}\right): n \in N\right\} \subset \mathbb{R}$.

Suppose $X_{0}=\bigcap\left\{G_{n}: n \in N\right\}$, where each $G_{n}$ is open in $X$. Denote the relative topology on $X_{0}$ by $\mathcal{T}_{0}$, and define $\gamma: \mathcal{T}_{0}^{n} \rightarrow \mathcal{T}$ so that $X_{0} \cap \gamma(U)=U$ for all $U$ in $\mathcal{J}_{0}$. Define, by induction, a sequence $\mathcal{S}_{0}=\left(\psi_{n}\right)_{n} \in N$ which satisfies (2.1) and (2.2) relative to $\left(X_{0}, \mathcal{J}_{0}\right)$, and such that if $n \in N$ and $\left(U_{1}, \cdots, U_{n}\right) \in D\left(\psi_{n}\right)$, then $\left(G_{1} \cap \gamma\left(U_{1}\right), \cdots, G_{n} \cap \gamma\left(U_{n}\right)\right) \in D\left(\phi_{n}\right)$ and

$$
\psi_{n}\left(U_{1}, \cdots, U_{n}\right)=X_{0} \cap \phi_{n}\left(G_{1} \cap \gamma\left(U_{1}\right), \cdots, G_{n} \cap \gamma\left(U_{n}\right)\right) .
$$

In detail: Suppose $n>1$, and that, for $j=1, \cdots, n-1, \psi_{j}$ has been defined. Suppose $\left(U_{1}, \cdots, U_{n}\right) \in\left(\mathcal{T}_{0}^{*}\right)^{n}$ and $U_{j+1} \subset \psi_{j}\left(U_{1}, \cdots, U_{j}\right)$ for $j=$ $1, \cdots, n-1$. Then

$$
\begin{aligned}
X_{0} \cap \gamma\left(U_{n}\right) & \subset \psi_{n-1}\left(U_{1}, \cdots, U_{n-1}\right) \\
& \subset \phi_{n-1}\left(G_{1} \cap \gamma\left(U_{1}\right), \cdots, G_{n-1} \cap \gamma\left(U_{n-1}\right)\right) .
\end{aligned}
$$

Since $R\left(\phi_{n-1}\right) \subset R$ and $X_{0}$ is dense in $X, \gamma\left(U_{n}\right)$ is contained in $\phi_{n-1}\left(G_{1} \cap \gamma\left(U_{1}\right), \cdots, G_{n-1} \cap \gamma\left(U_{n-1}\right)\right)$. Therefore $\psi_{n}\left(U_{1}, \cdots, U_{n}\right)$ can be defined by (3.1). 
Now, if $\left(U_{n}\right)_{n \in N}$ is an $\mathcal{S}_{0}$-sequence, then $\left(G_{n} \cap \gamma\left(U_{n}\right)\right)_{n \in N}$ is an $\mathcal{S}$ sequence. Hence $\bigcap\left\{U_{n}: n \in N\right\}=\bigcap\left\{G_{n} \cap \gamma\left(U_{n}\right): n \in N\right\} \neq \varnothing$, and $\mathcal{S}_{0}$ is a winning strategy for $\left(X_{0}, \mathcal{T}_{0}\right)$.

(7) Define $\theta^{*}: \mathcal{J}^{*} \rightarrow \mathcal{U}^{*}$ by letting $\theta^{*}(U)=Y \sim \theta[X \sim U]$ for each $U$ in $\mathcal{J}^{*}$.

If $(X, \mathcal{T})$ is weakly $\alpha$-favorable and $\mathcal{S}=\left(\phi_{n}\right)_{n \in N}$ is a winning strategy for $(X, \mathcal{T})$, then we define, by induction, a sequence $\mathcal{S}_{0}=\left(\psi_{n}\right)_{n \in N}$ which satisfies (2.1) and (2.2) relative to $(Y, \mathcal{U})$ and such that, if $n \in N$ and $\left(V_{1}, \cdots, V_{n}\right) \in D\left(\psi_{n}\right)$, then $\left(\cap^{-1}\left[V_{1}\right], \cdots, \Omega^{-1}\left[V_{n}\right]\right) \in D\left(\phi_{n}\right)$ and

$$
\psi_{n}\left(V_{1}, \cdots, V_{n}\right)=\theta^{*}\left(\phi_{n}\left(\theta^{-1}\left[V_{1}\right], \cdots, \theta^{-1}\left[V_{n}\right]\right)\right) \text {. }
$$

If $\left(V_{n}\right)_{n \in N}$ is an $\mathcal{S}_{0}$-sequence, then $\left(\theta^{-1}\left[V_{n}\right]\right)_{n \in N}$ is an $\mathcal{S}$-sequence, so $\bigcap\left\{V_{n}: n \in N\right\}=\theta\left[\bigcap\left\{\theta^{-1}\left[V_{n}\right]: n \in N\right\}\right] \neq \varnothing$.

If $(Y, \mathcal{U})$ is weakly $\alpha$-favorable and $\mathcal{S}_{0}=\left(\psi_{n}\right)_{n \in N}$ is a winning strategy for $(Y, \mathcal{U})$, then we define, by induction, a sequence $\mathcal{S}=\left(\phi_{n}\right)_{n \in N}$ which satisfies (2.1) and (2.2) and such that, if $n \in N$ and $\left(U_{1}, \cdots, U_{n}\right) \in D\left(\phi_{n}\right)$, then $\left(\theta^{*}\left(U_{1}\right), \cdots, \theta^{*}\left(U_{n}\right)\right) \in D\left(\psi_{n}\right)$ and

$$
\phi_{n}\left(U_{1}, \cdots, U_{n}\right)=\theta^{-1}\left[\psi_{n}\left(\theta^{*}\left(U_{1}\right), \cdots, \theta^{*}\left(U_{n}\right)\right)\right] .
$$

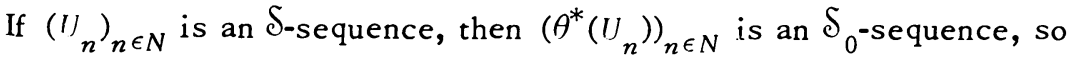

$$
\bigcap\left\{U_{n}: n \in N\right\} \supset \bigcap\left\{\theta^{-1}\left[\theta^{*}\left(U_{n}\right)\right]: n \in N\right\}=\theta^{-1}\left[\bigcap\left\{\theta^{*}\left(U_{n}\right): n \in N\right\}\right] \neq \varnothing .
$$

(8) Suppose $\mathcal{S}=\left(\phi_{n}\right)_{n \in N}$ is a winning strategy for $(X, \mathcal{T})$. Define, by induction, sequences $\mathcal{S}_{0}=\left(\psi_{n}\right)_{n \in N},\left(\gamma_{n}\right)_{n \in N}$ of functions such that (a) $\mathcal{S}_{0}$ satisfies (2.1) and (2.2) relative to $(Y, \mathcal{U}),(\mathrm{b}) D\left(\gamma_{1}\right)=D\left(\psi_{1}\right)$ and, if $V \in D\left(\gamma_{1}\right)$, then $\gamma_{1}(V)=\theta^{-1}[V]$ and $\psi_{1}(V)=\theta\left[\phi_{1}\left(\gamma_{1}(V)\right)\right]$, and (c) for all $n$ in $N$, (n.1) $D\left(\gamma_{n+1}\right)=D\left(\psi_{n+1}\right)$ and $R\left(\gamma_{n+1}\right) \subset D\left(\phi_{n+1}\right)$, and (n.2) if $\left(V_{1}, \cdots, V_{n+1}\right) \in D\left(\gamma_{n+1}\right)$ and $\gamma_{n}\left(V_{1}, \cdots, V_{n}\right)=\left(U_{1}, \cdots, U_{n}\right)$, then

$$
\gamma_{n+1}\left(V_{1}, \cdots, V_{n+1}\right)=\left(U_{1}, \cdots, U_{n}, \theta^{-1}\left[V_{n+1}\right] \cap \phi_{n}\left(U_{1}, \cdots, U_{n}\right)\right)
$$

and

$$
\psi_{n+1}\left(V_{1}, \cdots, V_{n+1}\right)=\theta\left[\phi_{n+1}\left(\gamma_{n+1}\left(V_{1}, \cdots, V_{n+1}\right)\right)\right] .
$$

Now, if $\left(V_{n}\right)_{n \in N}$ is an $\mathcal{S}_{0}$-sequence, then there is an $\mathcal{S}_{\text {-sequence }}$ $\left(U_{n}\right)_{n \in N}$ such that $\theta\left[U_{n}\right]=V_{n}$ for all $n$ in $N$. Hence $\bigcap\left\{V_{n}: n \in N\right\} \supset$ $\theta\left[\bigcap\left\{U_{n}: n \in N\right\}\right] \neq \varnothing$.

(9) Suppose $\mathcal{S}=\left(\phi_{n}\right)_{n \in N}$ is a winning strategy for $(Y, U)$ and that 
$\left(G_{n}\right)_{n \in N}$ is a sequence of dense, open subsets of $X \times Y$. It suffices to show that $\bigcap\left\{G_{n}: n \in N\right\} \neq \varnothing$.

We define, by induction, a sequence $\left(\gamma_{n}\right)_{n \in N}$ of functions such that, for each $n$ in $N$, (n.1) $D\left(\gamma_{n}\right)$ is a disjoint subfamily of $\mathcal{T}^{*}, R\left(\gamma_{n}\right) \subset \mathcal{U}^{*}$, and $H \times \gamma_{n}(H) \subset G_{n}$ for all $H$ in $D\left(\gamma_{n}\right),\left(\right.$ n.2) $D\left(\gamma_{n+1}\right)$ refines $D\left(\gamma_{n}\right),($ n.3) if $H_{j} \in D\left(\gamma_{j}\right)$ for $j=1, \cdots, n$, and $H_{j+1} \subset H_{j}$ for $j=1, \cdots, n-1$, then $\left(\gamma_{1}\left(H_{1}\right)\right.$, $\left.\cdots, \gamma_{n}\left(H_{n}\right)\right) \in D\left(\phi_{n}\right)$, and (n.4) $\bigcup D\left(\gamma_{n}\right)$ is dense in $X$. In detail: At the $n$th step, let $\widetilde{F}_{n}$ denote the set of all functions $\gamma$ such that (n.1) and (n.3) (and, if $n>1,((n-1) .2))$, with $\gamma_{n}$ replaced by $\gamma$, hold. Order $\mathcal{F}_{n}$ by inclusion. Since Zorn's lemma is applicable, there is a maximal element $\gamma_{n}$ in $\mathcal{F}_{n}$. If $\gamma_{n}$ does not satisfy (n.4) and $n>1$, then, for $j=1, \cdots, n-1$, there is $H_{j}$ in $D\left(\gamma_{j}\right)$ such that $H_{j+1} \subset H_{j}$ for $j=1, \cdots, n-2$, and $H_{n-1} \cap[X \sim$ $\left.\operatorname{cl}\left[\bigcup D\left(\gamma_{n}\right)\right]\right] \neq \varnothing$. So there are $H$ in $\mathcal{T}^{*}, K$ in $\mathcal{U}^{*}$ such that $H \times K$ is contained in

$$
G_{n} \cap\left\{\left[H_{n-1} \cap\left[X \sim \mathrm{cl}\left[\bigcup D\left(\gamma_{n}\right)\right]\right]\right] \times \phi_{n-1}\left(\gamma_{1}\left(H_{1}\right), \cdots, \gamma_{n-1}\left(H_{n-1}\right)\right)\right\} .
$$

Then $\gamma_{n} \cup(H, K) \in \mathcal{F}_{n}$. Therefore $\gamma_{n}$ satisfies (n.4).

Since $X$ is a Baire space, $\bigcap\left\{\bigcup D\left(\phi_{n}\right): n \in N\right\} \neq \varnothing$. Suppose $x \in$ $\cap\left\{\bigcup D\left(\phi_{n}\right): n \in N\right\}$. Then there is a sequence $\left(H_{n}\right)_{n \in N}$ such that for each $n$ in $N, H_{n} \in D\left(\gamma_{n}\right)$ and $H_{n+1} \subset H_{n}$. By $((\mathrm{n} .3))_{n \in N},\left(\gamma_{n}\left(H_{n}\right)\right)_{n \in N}$ is an $\mathcal{S}$ sequence; hence $\bigcap\left\{\gamma_{n}\left(H_{n}\right): n \in N\right\} \neq \varnothing$. And, if $y \in \bigcap\left\{\gamma_{n}\left(H_{n}\right): n \in N\right\}$, then $(x, y) \in \bigcap\left\{H_{n} \times \gamma_{n}\left(H_{n}\right): n \in N\right\} \subset \bigcap\left\{G_{n}: n \in N\right\}$.

(10) Suppose $\mathcal{S}=\left(\phi_{n}\right)_{n \in N}$ is a winning strategy for $(X, \mathcal{J})$ and $\mathcal{P}=$ $\bigcup\left\{\mathcal{P}_{n}: n \in N\right\}$ where, for each $n$ in $N, \mathscr{P}_{n}$ is a disjoint family and $\bigcup \mathcal{P}_{n}$ is dense in $X$.

We define, by induction, a sequence $\left(\gamma_{n}\right)_{n \in N}$ of functions such that, for each $n$ in $N$, (n.1) $D\left(\gamma_{n}\right)$ is a disjoint subfamily of $\mathcal{T}^{*}$ that refines $\mathfrak{P}_{n}$ and $R\left(\gamma_{n}\right) \subset \mathcal{T}^{*},\left(\right.$ n.2) $D\left(\gamma_{n+1}\right)$ refines $D\left(\gamma_{n}\right),\left(\right.$ n.3) if $H_{j} \in D\left(\gamma_{j}\right)$ for $j=1$, $\cdots, n$, and $H_{j+1} \subset H_{j}$ for $j=1, \cdots, n-1$, then $\left(\gamma_{1}\left(H_{1}\right), \cdots, \gamma_{n}\left(H_{n}\right)\right) \epsilon$ $D\left(\phi_{n}\right)$ and $\phi_{n}\left(\gamma_{1}\left(H_{1}\right), \cdots, \gamma_{n}\left(H_{n}\right)\right)=H_{n}$, and (n.4) $D\left(\gamma_{n}\right)$ is dense in $X$. In detail: At the $n$th step, let $\gamma_{n}$ be a maximal element of $\mathcal{F}_{n}$, where we define $\mathcal{F}_{n}$ verbatim as in (9). If $\gamma_{n}$ does not satisfy (n.4) and $n>1$, then there is $P_{n}$ in $\mathcal{P}_{n}$ and, for $j=1, \cdots, n-1$, there is $H_{j}$ in $D\left(\gamma_{j}\right)$ such that $H_{j+1} \subset H_{j}$ for $j=1, \cdots, n-2$ and

$$
K=P_{n} \cap H_{n-1} \cap\left[X \sim \mathrm{cl}\left[\bigcup D\left(\gamma_{n}\right)\right]\right] \neq \varnothing .
$$


Then

$$
\gamma_{n} \cup\left\{\left(\phi_{n}\left(\gamma_{1}\left(H_{1}\right), \cdots, \gamma_{n-1}\left(H_{n-1}\right), K\right), K\right)\right\} \in \mathcal{F}_{n} .
$$

Therefore $\gamma_{n}$ satisfies (n.4).

Let $X_{0}=\bigcap\left\{\bigcup D\left(\gamma_{n}\right): n \in N\right\}$ and $B_{0}=\left\{H \cap X_{0}: H \in \bigcup\left\{D\left(\gamma_{n}\right): n \in N\right\}\right\}$. Then $\Re_{0}$ is a base for a topology $\mathcal{T}_{0}$ on $X_{0}$. Define the pseudo-metric $d$ on $X_{0}$ by letting

$$
d(x, y)=\inf \left\{n^{-1}: \text { there is } H_{n} \text { in } D\left(\gamma_{n}\right) \text { such that } x, y \in H_{n}\right\}
$$

for all $x, y$ in $X_{0}$. Then $\mathcal{T}_{0}$ is the pseudo-metric topology induced by $d$. In fact, if $H_{n} \in D\left(\gamma_{n}\right)$ and $x \in H_{n} \cap X_{0}$, then $\left\{y \in X_{0}: d(x, y) \leq n^{-1}\right\}=$ $H_{n} \cap X_{0^{*}}$ And $\left(X_{0}, d\right)$ is complete. For suppose $\left(x_{n}\right)_{n \in N}$ is a Cauchy sequence in $\left(X_{0}, d\right)$. We may assume that, for all $n$ in $N, d\left(x_{n}, x_{n+1}\right) \leq n^{-1}$. Then there is a sequence $\left(H_{n}\right)_{n \in N}$ such that, for all $n$ in $N, x_{n} \in H_{n} \in D\left(\gamma_{n}\right)$ and $H_{n+1} \subset H_{n}$. By $((\mathrm{n} .3))_{n \in N},\left(\gamma_{n}\left(H_{n}\right)\right)_{n \in N}$ is an S-sequence; hence $\bigcap\left\{\gamma_{n}\left(H_{n}\right): n \in N\right\} \neq \varnothing$. But, since $\gamma_{n+1}\left(H_{n+1}\right) \subset H_{n}$ for each $n, \bigcap\left\{H_{n}\right.$ : $n \in N\} \neq \varnothing$. And, if $x \in \bigcap\left\{H_{n}: n \in N\right\}$, then $\left(x_{n}\right)_{n \in N}$ converges to $x$. Hence $\left(X_{0}, \mathcal{T}_{0}\right)$ is both $\alpha$-favorable and pseudo-complete. Since $\mathcal{T}_{0}$ is a pseudobase for the relative topology $\mathcal{T}\left(X_{0}\right)$ on $X_{0},\left(X_{0}, \mathcal{T}\left(X_{0}\right)\right)$ is both $\alpha$-favorable and pseudo-complete. Since $X_{0}$ is dense in $X,(X, \mathfrak{T})$ is $\alpha$-favorable and, if $\mathcal{T}$ is quasi-regular, $(X, \mathcal{J})$ is pseudo-complete.

(11) The proof of (11) is very similar to the proof of (10). In fact, if $(X, \mathcal{J})$ is a $T_{0}$ space without any isolated points, and $\mathcal{B}$ is a base of countable order for $\mathcal{T}$, then we can choose the sequence $\left(\mathcal{P}_{n}\right)_{n \in N}$ so that $\bigcup\left\{\mathcal{P}_{n}\right.$ : $n \in N\} \subset B_{\text {and }} \mathscr{P}_{n} \cap \mathcal{P}_{n+1}=\varnothing$ for all $n$ in $N$. Then it is easily verified that $\mathcal{T}_{0}=\mathcal{T}\left(X_{0}\right)$; hence $X_{0}$ is the required metrically topologically complete dense $G_{\delta}$.

\section{REFERENCES}

1. J. M. Aarts and D. J. Lutzer, Completeness properties designed for recognizing Baire spaces (to appear).

2. - Pseudo-completeness and the product of Baire spaces, Pacific J. Math. 48 (1973), 1-10.

3. G. Choquet, Lectures on analysis. I: Integration and topological vector spaces, Benjamin, New York, 1969. MR $40 \# 3252$.

4. J. C. Oxtoby, Cartesian products of Baire spaces, Fund. Math. 49 (1960/61), 157-166. MR 25 \#4055; erratum, 26, 1453.

5. H. H. Wicke and J. M. Worrell, Jr., Open continuous mappings of spaces having bases of countable order, Duke Math. J. 34 (1967), 255-272. MR 35 \#979. 